\title{
DRAFT

\section{NON-DESTRUCTIVE EVALUATION (NDE) OF SPACE APPLICATION PANELS USING PIEZOELECTRIC WAFER ACTIVE SENSORS}

\author{
Adrian Cuc \\ University of South Carolina \\ 300 S Main St Room A236 \\ Columbia, SC 29208 \\ Phone: (803) 777-0619, Fax: (803) 777-0106 \\ E-mail: aicuc000@engr.sc.edu \\ Dr. Victor Giurgiutiu \\ University of South Carolina \\ 300 S Main St Room A222 \\ Columbia, SC 29208 \\ Phone: (803) 777-8018, Fax: (803) 777-0106 \\ E-mail: victorg@sc.edu
}

\author{
Zeb Tidwell \\ NextGen Aeronautics, Inc. \\ 2780 Skypark Dr., Suite 400 \\ Torrance, CA 90505 \\ Phone: (310) 891-2807 \\ ztidwell@yahoo.com \\ Dr. Shiv Joshi \\ NextGen Aeronautics, Inc. \\ 2780 Skypark Dr., Suite 400 \\ Torrance, CA 90505 \\ Phone: (310) 891-2807 \\ sjoshi@nextgenaero.com
}

\begin{abstract}
Ultrasonic guided waves inspection using Lamb waves is suitable for damage detection in metallic structures. This paper will present experimental results obtained using guided Lamb waves to detect flaws in aluminum specimens with design features applicable to space applications. Two aluminum panels were fabricated from a variable-thickness aluminum top plate, with two bolted I-beams edge stiffeners and four bonded angle stiffeners. Artificial damages were introduced in the two panels: cracks, corrosions, and disbonds. The proposed investigation methods used embedded piezoelectric wafer active sensors (PWAS) to excite and receive Lamb waves. Three wave propagation methods were used: pitch-catch, pulseecho, and the embedded ultrasonic structural radar (EUSR). In addition, we also used a standing-wave damage detection technique, the electro-mechanical impedance method. The paper will present in detail the salient results from using these methods for damage detection and structural health monitoring. Where appropriate, comparison between different methods in detecting the same damage will be performed.

The results have demonstrated the ability of piezoelectric wafer active sensors working in conjunction with guided Lamb waves to detect various types of damages present in complex geometry structures typical of space applications.
\end{abstract}

Keywords: NDE, ultrasonic, Lamb waves, crack, corrosion, disbond, PWAS

\section{INTRODUCTION}

Structural health monitoring (SHM) is an emerging research area with multiple applications. SHM assesses the state of structural health and, through appropriate data processing and interpretation, predicts the remaining life of the structure. There are many ultrasonic nondestructive evaluation (NDE), non-destructive inspection (NDI), and non-destructive testing (NDT) techniques for identifying local damage and detect flaws in metallic structures. Ultrasonic NDE methods rely on elastic wave propagation and reflection within the material. They try to identify the wave field disturbances due to local damage and flaws. Ultrasonic testing involves one or more of the following measurements: time of flight (TOF; wave transit or delay), path length, frequency, phase angle, amplitude, impedance, and angle of wave deflection (reflection and refraction). Conventional ultrasonic methods include the pulse-echo, the pitch-catch (or pulse-transmission), and the pulse-resonance techniques.

This paper will investigate the possibility of using embedded ultrasonic non-destructive evaluation and the opportunity for developing embedded structural health monitoring. SHM determines the health of a structure by readings an array of sensors that are embedded (permanently attached) into the structure and monitored over time. SHM can be either passive or active. Passive SHM infers the state of the structure using passive sensors that are monitored over time and fed into a structural model. Active SHM uses active sensors that interrogate the structure to detect the presence of damage, 
and to estimate its extent and intensity. One active SHM method employs piezoelectric wafer active sensors (PWAS), which send and receive ultrasonic Lamb waves and determine the presence of cracks, delaminations, disbonds, and corrosion. Two approaches are being considered: (a) traveling waves; and (b) standing waves.

\section{SPACECRAFT PANELS}

Two aluminum test panels were fabricated by NextGen Aeronautics, Inc. The panels consist of the skin (Al 7075, $24 \times 23.5 \times 0.125$ in) with a 3 in diameter hole in the center, two spars (Al 6061 I-beams, $3 \times 2.5 \times 0.250$ in and 24 in length), four stiffeners (Al 6063, 1x1x0.125 in and 18.5 in length) and fasteners installed from the skin side (Figure 1).

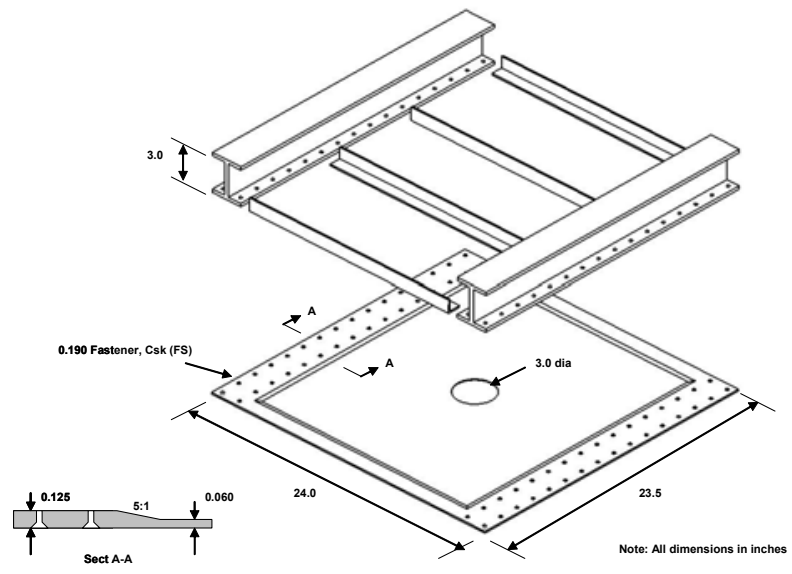

Figure 1 Structural panel design

The stiffeners were bonded to the aluminum skin using a structural adhesive, Hysol EA 9394. Damages were artificially introduced in the two specimens including cracks (CK), corrosions (CR), disbonds (DB), and cracks under bolts (CB). A summary of the type and size of the damages is provided in Table 1.

Table 1 Induced panel damages

\begin{tabular}{|c|l|c|c|}
\hline \multicolumn{2}{|c|}{ Panel 1 } & \multicolumn{2}{c|}{ Panel 2 } \\
\hline ID & \multicolumn{1}{|c|}{ Size (inches) } & ID & Size (inches) \\
\hline CK1 & $0.75 \times 0.015 \times$ thru thickness & CB1 & $3.70 \times 0.015 \times$ thru thickness \\
\hline CK2 & $0.50 \times 0.015 \times$ thru thickness & CB2 & $0.35^{*} \times 0.015 \times$ thru thickness \\
\hline CK3 & $0.50 \times 0.015 \times$ thru thickness & CB3 & $0.70 \times 0.015 \times$ thru thickness \\
\hline CK4 & $0.50 \times 0.015 \times$ thru thickness & CB4 & $0.35^{*} \times 0.015 \times$ thru thickness \\
\hline CR1 & $1.65 \times 0.38 \times 0.025$ & CB5 & $0.70 \times 0.015 \times$ thru thickness \\
\hline CR2 & $0.50 \times 0.38 \times 0.025$ & CB6 & $0.70 \times 0.015 \times$ thru thickness \\
\hline DB1 & $2.00 \times 0.50$ & CR1 & $2.30 \times 0.50 \times 0.025$ \\
\hline DB2 & $1.00 \times 1.00$ & CR2 & $2.00 \times 0.50 \times 0.025$ \\
\hline DB3 & $2.00 \times 0.50$ & & \\
\hline DB4 & $1.00 \times 1.00$ & & \\
\hline
\end{tabular}

A schematic of the aluminum Panel 1 specimen showing the location of the damage is presented in Figure 2. Panel 1 contains disbonds, cracks and corrosions. The disbonds are located between the stiffeners and the skin. They are of two types: partial disbonds DB1 and DB3, and through disbonds
DB2 and DB4. The corrosions are simulated as machined areas were part of material was removed. The four cracks presented are in the shape of a slit and are through cracks located on the skin of the panel.

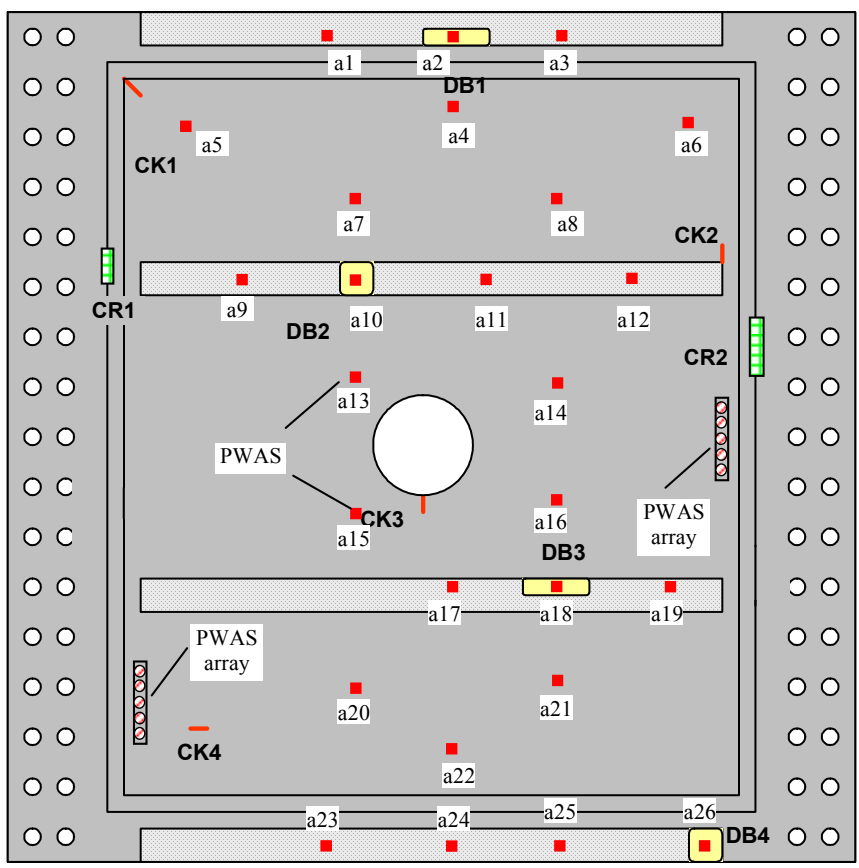

Figure 2 Schematic of the location and the type of the damage on the Panel 1 specimen (top view)

The schematic of Panel 2 is presented in Figure 3. Panel 2 contains only corrosion and cracks. The two corrosions are hidden between the skin and the spars and are simulated in the similar way of removing material. Cracks are located under the bolts and are through the thickness cracks.

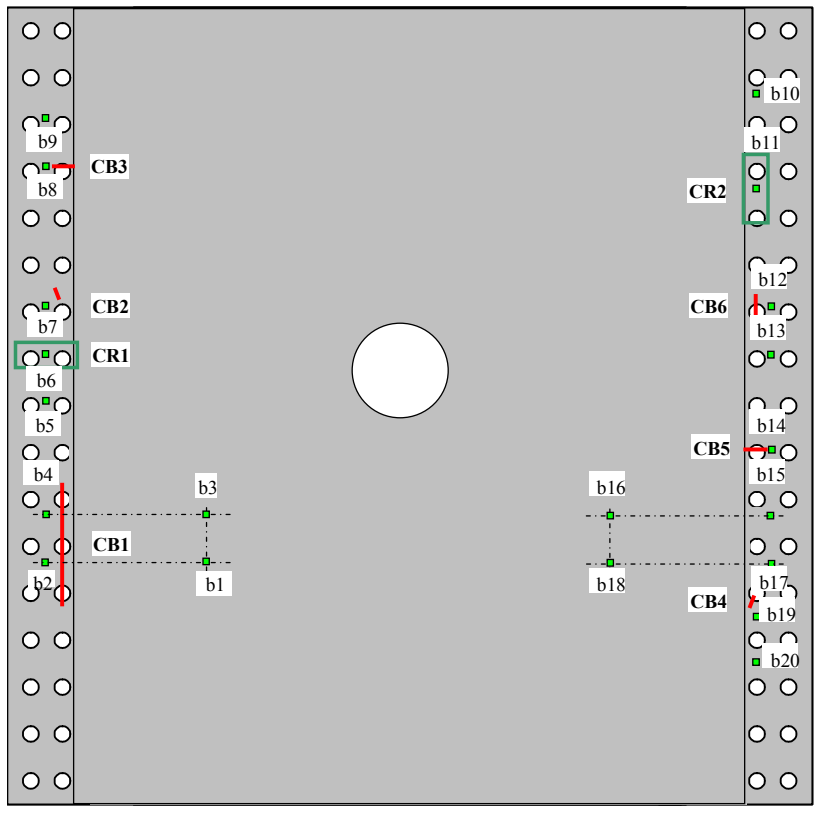

Figure 3 Schematic of the location and the type of the damage on the Panel 2 specimen (bottom view) 
The two panels were instrumented with piezoelectric wafer active sensors (PWAS) as shown in Figure 2 and Figure 3. The PWAS are used for both sensing and receiving Lamb waves. The experimental set-up is presented in Figure 4.
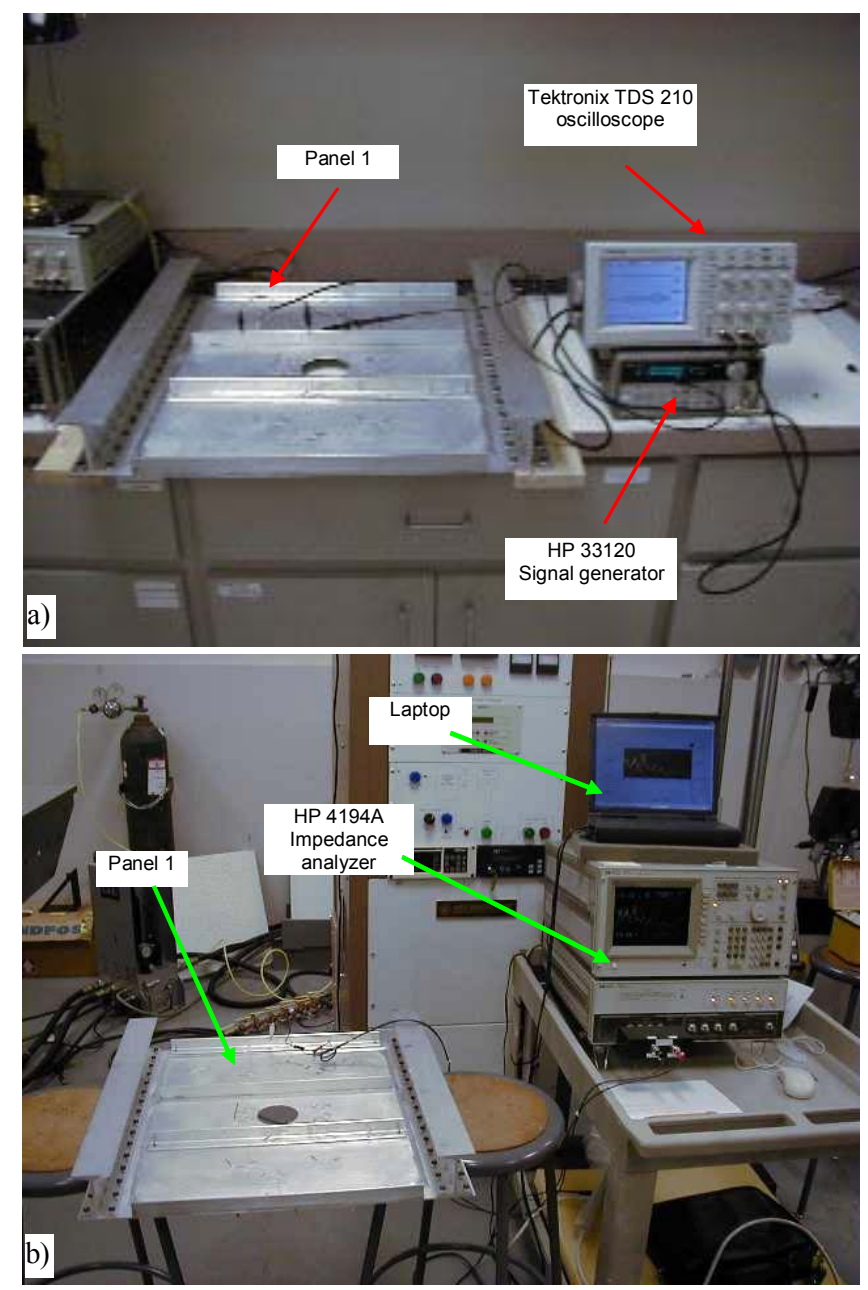

Figure 4 Instrumentation set-up: (a) wave propagation; (b) electromechanical impedance

The instrumentation set-up for the wave propagation method is shown in Figure 4a. An HP 33120 signal generator was used to excite the PWAS with a 3-count sinusoidal burst signal at a frequency of $330 \mathrm{kHz}$. The received signal was displayed on a Tektronix TDS 210 digital oscilloscope and transferred to a laptop. For the electromechanical impedance method the instrumentation set-up is shown in Figure 4b. The real part of the EM impedance spectrum was recorded using an HP 4194A impedance analyzer, and the frequency range of the excitation was from $150 \mathrm{kHz}$ to $1.5 \mathrm{MHz}$.

\section{DETECTION STRATEGIES}

Guided waves can be generated and detected using several methods. In conventional ultrasonic methods guided waves are generated using large ultrasonic transducers that are usually positioned at an angle through a wedge. Another method to create guided waves is to use comb transducers. The comb spacing is such that it tunes with the guided half-wavelength.

The method proposed and used in this experiment to excite and receive guided waves uses small piezoelectric wafer sensors
(PWAS) permanently attached to the structure ${ }^{[1,2]}$. PWAS are thin piezoelectric ceramic wafers, round or rectangular in shape, and are small, lightweight and inexpensive compared to traditional ultrasonic transducers. PWAS operate on the piezoelectric principle that couples the electrical and mechanical variables in the material (mechanical strain, Sij, mechanical stress, Tkl, electrical field, Ek, and electrical displacement $\mathrm{Dj}$ ) in the form:

$$
\begin{aligned}
& S_{i j}=s_{i j k l}^{E} T_{k l}+d_{k i j} E_{k} \\
& D_{j}=d_{j k l} T_{k l}+\varepsilon_{j k}^{T} E_{k}
\end{aligned}
$$

where $S_{i j k l}^{E}$ is the mechanical compliance of the material measured at zero electric field $(\mathrm{E}=0), \varepsilon_{j k}^{T}$ is the dielectric permittivity measured at zero mechanical stress $(\mathrm{T}=0)$, and $d_{k i j}$ represents the piezoelectric coupling effect. The direct piezoelectric effect is when the applied stress on the sensor is converted into an electric charge. The inverse effect, conversely, will produce strain when a voltage is applied on the sensor. In this way the PWAS can be used as both, a transmitter and a receiver. For embedded NDE applications, PWAS couple their in-plane motion, excited by the applied oscillatory voltage through the piezoelectric effect, with the Lamb-waves particle motion on the material surface. Lamb waves can be either quasi-axial (S0, S1, S2, ..), or quasi-flexural (A0, A1, A2, ..). An important characteristic of PWAS, which distinguishes them from conventional ultrasonic transducers, is their capability of tuning into various guided wave modes. Moulin et al. (1997) were among the first to realize the generation of Lamb waves with an embedded piezoelectric disc. Using finite element modeling and experimental results, they showed the excitation of S0, S1, A0, A1, and A2 Lamb waves at up to 2.5 $\mathrm{MHz}$ frequencies. Lin and Yuan (2001a) studied the use of piezoceramic discs for the generation and detection of circularcrested flexural waves in a composite plate using the Mindlin theory. The solution was derived in terms of Henkel functions. Propagation of smoothed tone-bursts was studied. The theoretical predictions compared favorably with experimental measurements. Liu et al. (2003) investigated the input-output characteristic of piezoelectric structural health monitoring systems for composite plates. Lee and Staszewski (2003) used finite differences and local interaction simulation approach (LISA) to model the generation of Lamb waves with surfacemounted piezoceramic wafers for the detection of structural damage. Numerical simulation compared favorably with experiments. Qing et al. (2003) reported experimental results indicating that PWAS effectiveness in exciting A0 and S0 Lamb modes varies significantly with the PWAS location across the laminate thickness.

Although a complete modeling of this interaction is not yet available, some simplified models exist that clarify the underlying principles of Lamb wave tuning with PWAS transducer. Giurgiutiu (2003) developed a plane-strain analysis of the PWAS-structure interaction using the space-domain Fourier analysis. This model illustrates the principles of PWAS Lamb-wave mode tuning, and opens the path for a more comprehensive analysis.

A significant number of sensors were initially used to ensure a good signal response near known damage locations. The optimum number of sensors/methods is to be determined such that the signal response will give an acceptable probability 
of detection for certain types of damage. The detection capabilities used are:

- Wave propagation

○ Pitch-catch: disbond detection

- Pulse-echo: disbond and crack detection

- Embedded Ultrasonic Structural Radar (EUSR): crack detection

- Standing wave

- Electromechanical Impedance (EMI): disbond, crack and corrosion detection

The detection methods used in this experiment are summarized in Table2. This table provides a quick correlation between the damage type and the detection method.

Table 2 Detection capabilities of proposed methods

\begin{tabular}{|c|c|c|c|c|c|c|}
\hline \multicolumn{3}{|c|}{ Method } & \multicolumn{3}{|c|}{ Wave propagation } & \multirow{3}{*}{$\begin{array}{c}\begin{array}{c}\text { Standing } \\
\text { waves }\end{array} \\
\text { EMI } \\
\mathrm{x}\end{array}$} \\
\hline \multicolumn{3}{|c|}{ Damage } & Pitch- & Pulse- & EUSR & \\
\hline \multirow{10}{*}{$\begin{array}{l}\bar{\Xi} \\
\bar{\Xi} \\
\tilde{\Xi}\end{array}$} & \multirow{4}{*}{ Disbond } & DB1 & $\mathrm{x}$ & & & \\
\hline & & DB2 & $\mathrm{x}$ & $\mathrm{x}$ & & \\
\hline & & DB3 & $\mathrm{x}$ & $\mathrm{x}$ & & \\
\hline & & DB4 & & & & $\mathrm{x}$ \\
\hline & \multirow{4}{*}{ Crack } & CK1 & & & $\mathrm{x}$ & $\mathrm{x}$ \\
\hline & & CK2 & & & & \\
\hline & & CK3 & & & $\mathrm{x}$ & \\
\hline & & CK4 & & & & \\
\hline & \multirow{2}{*}{ Corrosion } & CR1 & & & & $\mathrm{x}$ \\
\hline & & CR2 & & & & $\mathrm{x}$ \\
\hline \multirow{8}{*}{ 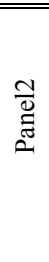 } & \multirow{6}{*}{ Crack } & CB1 & $\mathrm{x}$ & $\mathrm{x}$ & & $\mathrm{x}$ \\
\hline & & CB2 & & & & $\mathrm{x}$ \\
\hline & & CB3 & & & & $\mathrm{x}$ \\
\hline & & CB4 & & & & $\mathrm{x}$ \\
\hline & & CB5 & & & & $\mathrm{x}$ \\
\hline & & CB6 & & & & $\mathrm{x}$ \\
\hline & \multirow{2}{*}{ Corrosion } & CR1 & & & & $\mathrm{x}$ \\
\hline & & CR2 & & & & $x$ \\
\hline
\end{tabular}

A sample of the test matrix for data collection from the PWAS is presented in Table3:

Table 3 Test matrix

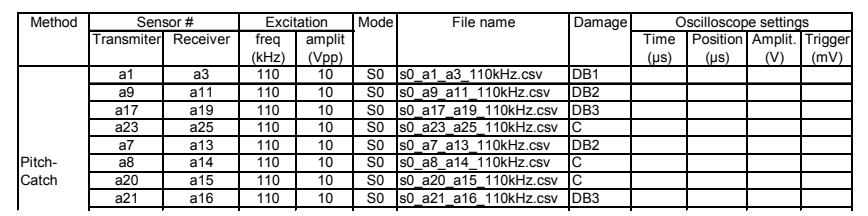

\section{Pitch-catch method}

The pitch-catch method can be used to detect structural changes that take place between a transmitter transducer and a receiver transducer. The detection is performed through the examination of the guided wave amplitude, phase, dispersion, and time of flight in comparison with a "pristine" situation. Guided wave modes that are strongly influenced by small changes in the material stiffness and thickness (such as the $A_{0}$ Lamb wave) are well suited for this method. Typical applications include: (a) corrosion detection in metallic structures; (b) diffused damage in composites; (c) disbond detection in adhesive joints; (d) delamination detection in layered composites, etc. Pitch-catch method can also be used to detect the presence of cracks from the wave signal diffracted by the crack.

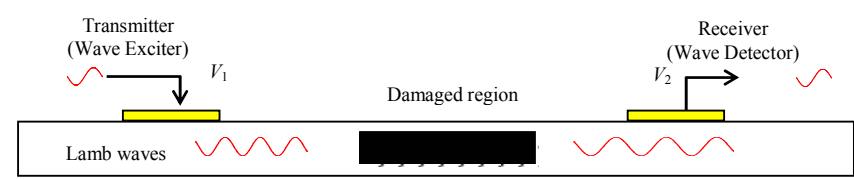

Figure 5 Embedded ultrasonics damage detection: pitchcatch method

The pitch-catch method detects damage from the changes that Lamb waves undergo when traveling through a damaged region. The method uses the transducers in pairs, one as transmitter, the other as receiver. In the embedded pitch-catch method (Figure 5), the transducers are either permanently attached to the structure or inserted between the layers of composite layup.

\section{Pulse-echo method}

In conventional NDE, the pulse echo method has traditionally been used for through-the-thickness testing. For large area inspection, through-the-thickness testing requires manual or mechanical moving of the transducer over the area of interest, which is labor intensive and time consuming. It seems apparent that guided-wave pulse echo seems more appropriate, since wide coverage could be achieved from a single location.

For crack-detection with the pulse-echo method, an appropriate Lamb-wave mode must be selected. Giurgiutiu et al (2003) used finite element simulation to show that the S0 Lamb waves can give much better reflections from through-the-thickness cracks than the A0 Lamb waves. This effect can be attributed to S0 being: (a) better reflected from the crack; and (b) much less dispersive. The first fact gives a strong signal, while the second ensures that the wave packet is compact and easy to interpret.

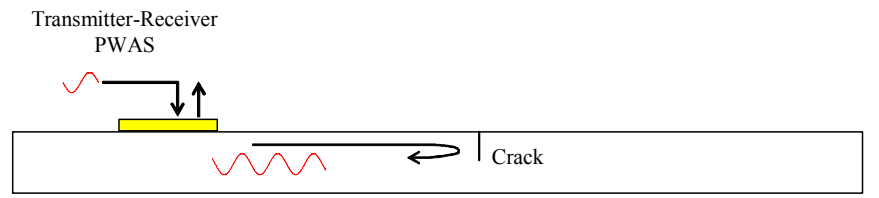

Figure 6 Embedded ultrasonics damage detection: pulseecho method

The use of Lamb-wave pulse echo methods with embedded PWAS follows the general principles of conventional Lambwave NDE. A PWAS transducer attached to the structure acts as both transmitter and detector of acoustic guided waves traveling in the structure. The wave sent by the PWAS is partially reflected at the crack. The echo is captured at the same PWAS acting as receiver (Figure 6). For the method to be successful, it is important that a low-dispersion Lamb wave is used. The selection of such a wave, e.g., the S0 mode, is achieved through the Lamb-wave tuning methods (Giurgiutiu, 2003)

\section{Embedded Ultrasonic Structural Radar (EUSR)}

Real-time phased array systems have become very popular in NDE practice. Giurgiutiu and Bao (2002) developed a PWAS phased array application and named it embedded ultrasonics structural radar (EUSR). Its principle of operation is derived from two general concepts:

(1) The guided Lamb wave generation with piezoelectric wafer active sensors (PWAS) 
(2) The principles of conventional phased-array radar.

The EUSR approach is different from the conventional phased array approach in two aspects:

1. It uses embedded transducers (PWAS)

2. It works in virtual time, not in real time.

Of these two, the latter is most important for structural health monitoring, because it permits the phased array benefits without the drawback of real-time multiple channel phasing. Whereas real-time phased-array transducers require heavy and complex multi-channel phasing equipment, the virtual-time approach adopted by the EUSR method can be done with only one channel and very simple equipment

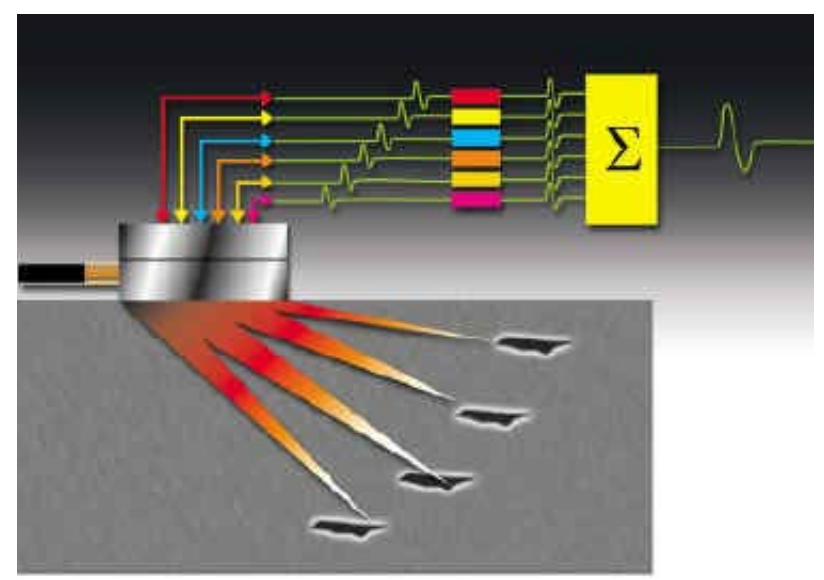

Figure 7 Conventional phased-array ultrasonic probe (Krautkramer, 2002)

The principles of conventional phased-array radar (Silvia, 1987) are applied to the EUSR algorithm assuming a uniform linear array of $M$ transmitter/receivers PWAS, where each PWAS acts as a point-wise omni-directional source/sink. The PWAS in the array are spaced at the distance $d=\lambda / 2$, which is assumed much smaller than the distance $r$ to a generic, fardistance point, P. Since $d<<r$, the rays joining the sensors with the point $\mathrm{P}$ can be assimilated with a parallel fascicle, of angle $\phi$. Therefore, for the $m^{\text {th }}$ PWAS, the distance will be shorted by $m(d \cos \phi)$. If all the PWAS are fired simultaneously, the signal from the $m^{\text {th }}$ PWAS will arrive at $\mathrm{P}$ quicker by $\Delta_{m}(\phi)=m(d \cos \phi) / c$. Yet, if the PWAS are not fired simultaneously, but with some individual delays, $\delta_{m}, m=$ $0,1, \ldots, M-1$, then the total signal received at point $\mathrm{P}$ will be:

$$
s_{\mathrm{P}}(t)=\frac{1}{\sqrt{r}} \sum_{m=0}^{M-1} s_{\mathrm{T}}\left(t-\frac{r}{c}+\Delta_{m}(\phi)-\delta_{m}\right)
$$

where $1 / \sqrt{r}$ represents the decrease in the wave amplitude due to the omni-directional 2-D radiation, and $r / c$ is the delay due to the travel distance between the reference PWAS $(m=0)$ and the point $\mathrm{P}$. (It is important to note that wave-energy conservation with no dissipation is assumed.)

\section{Electromechaincal Impedance method}

The impedance method is a damage detection technique complementary to the wave propagation techniques. The mechanical impedance method consists of exciting vibrations of bonded plates using a specialized transducer that simultaneously measures the applied normal force and the induced velocity.

The electro-mechanical impedance (EMI) method is an emerging technology that offers distinctive advantage over the mechanical impedance method. While the mechanical impedance method uses normal force excitation, the EM impedance method uses in-plane strain. The mechanical impedance transducer measures mechanical quantities (force and velocity/acceleration) to indirectly calculate the mechanical impedance, while the EM impedance active sensor measures the EM impedance directly as an electrical quantity. The principles of the EM impedance technique are illustrated in Figure 8

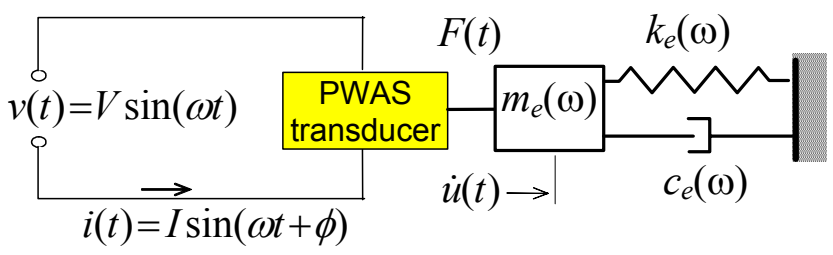

Figure 8 Embedded ultrasonics damage detection: electromechanical impedance method

The effect of a piezoelectric wafer active sensor affixed to the structure is to apply a local strain parallel to the surface that creates stationary elastic waves in the structure. The structure presents to the active sensor the drive-point impedance, $Z_{s t r}(\omega)=i \omega m_{e}(\omega)+c_{e}(\omega)-i k_{e}(\omega) / \omega$. Through the mechanical coupling between the PWAS and the host structure, on one hand, and through the electro-mechanical transduction inside the PWAS, on the other hand, the drive-point structural impedance is directly reflected into the effective electrical impedance as seen at the active sensor terminals. The apparent electro-mechanical impedance of the piezoelectric active sensor as coupled to the host structure is:

$$
Z(\omega)=\left[i \omega C\left(1-\kappa_{31}^{2} \frac{Z_{s t r}(\omega)}{Z_{P W A S}(\omega)+Z_{s t r}(\omega)}\right)\right]^{-1}
$$

where $Z(\omega)$ is the equivalent electromechanical admittance as seen at the PWAS terminals, $C$ is the zero-load capacitance of the PWAS, $\kappa_{31}$ is the electromechanical cross coupling coefficient of the PWAS $\left(\kappa_{31}=d_{13} / \sqrt{\overline{s_{11}} \bar{\varepsilon}_{33}}\right), Z_{\text {str }}$ is the impedance of the structure, and $Z_{\mathrm{PWAS}}$ is the impedance of the PWAS. The electromechanical impedance method is applied by scanning a predetermined frequency range in the hundreds of $\mathrm{kHz}$ band and recording the complex impedance spectrum. By comparing the impedance spectra taken at various times during the service life of a structure, meaningful information can be extracted pertinent to structural degradation and the appearance of incipient damage. It must be noted that the frequency range must be high enough for the signal wavelength to be significantly smaller than the defect size. 


\section{EXPERIMENTAL RESULTS}

\section{Pitch-catch method}

The PWAS used for the pitch-catch method and the actual location of the sensors used on Panel 1 is presented in Figure 9
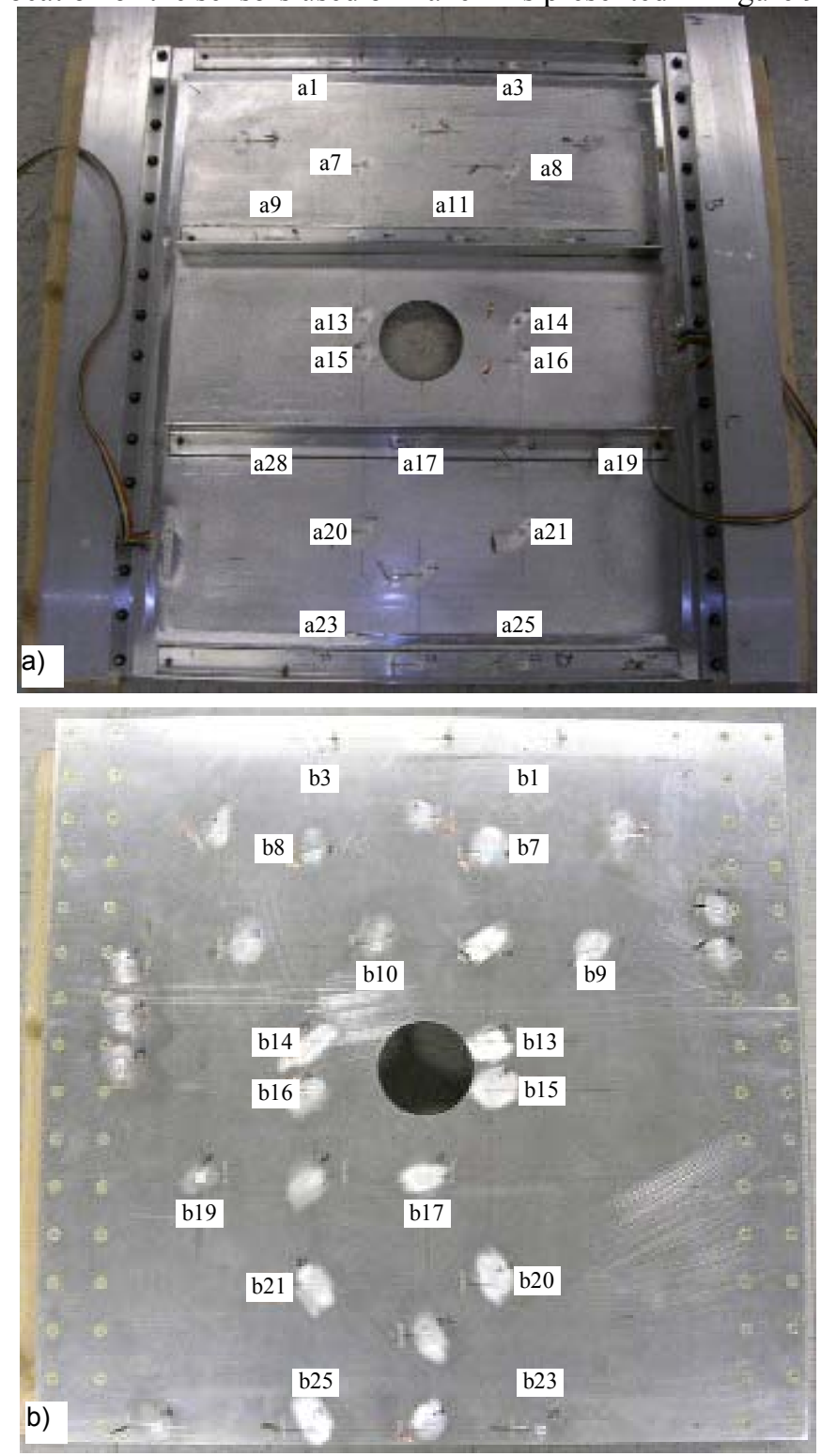

Figure 9 PWAS location on Panel 1 for pitch-catch tests: (a) top face of Panel 1; (b) bottom face of Panel 1

The results for the disbonds DB1, DB2, and DB3 are presented in Figure 10 through Figure 12. They show the signals sent and received from sensors located on a stiffener with a disbond and the signal received by a sensor located on a stiffener without a disbond.
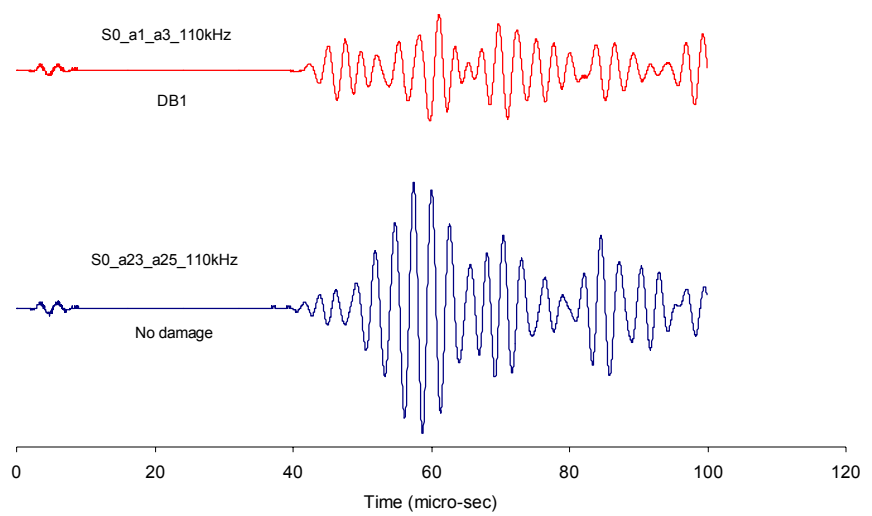

Figure 10 Pitch-catch method: the signal traveling over the disbond DB1 is compared with the signal traveling the same distance over a bonded area.
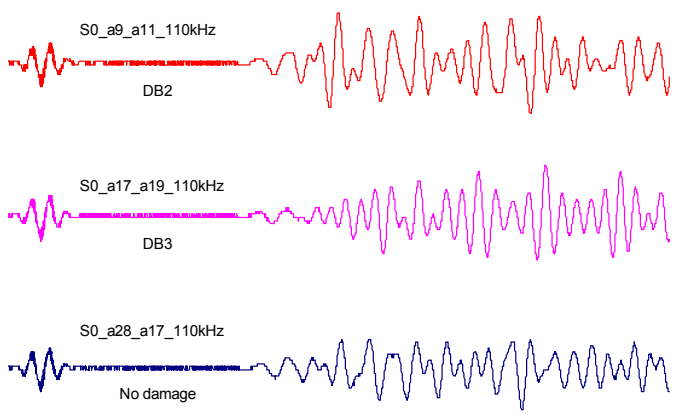

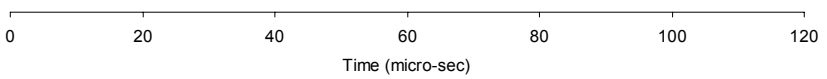

Figure 11 Pitch-catch method: the signals traveling over the disbond DB2 and DB3 are compared with the signal traveling the same distance over a bonded area

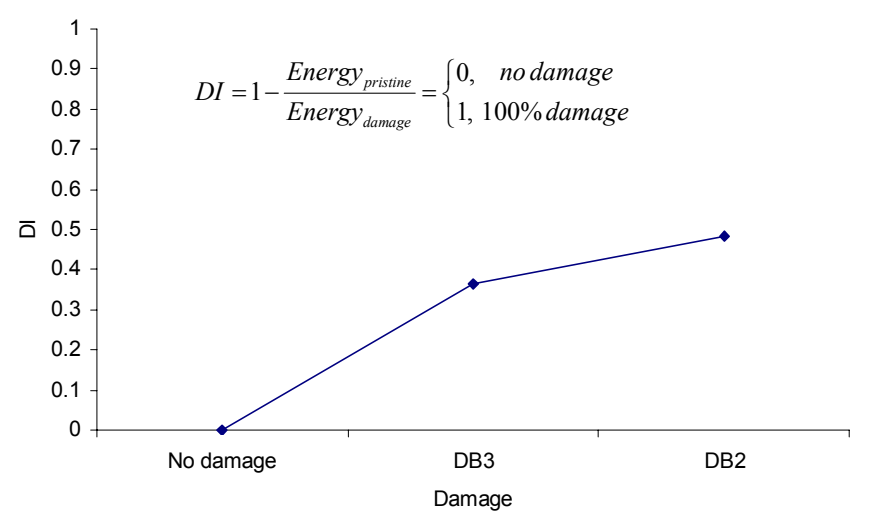

Figure 12 Damage Index of the three signals showing an increase of the DI with the severity of the damage

It can be seen from the pictures that there is a strong difference between the wave traveling over a disbond and the same wave traveling over a good bond. 


\section{Pulse-echo method}

The PWAS used for the pulse-echo method and the actual location of the sensors used on Panel 1 is presented in Figure 13.
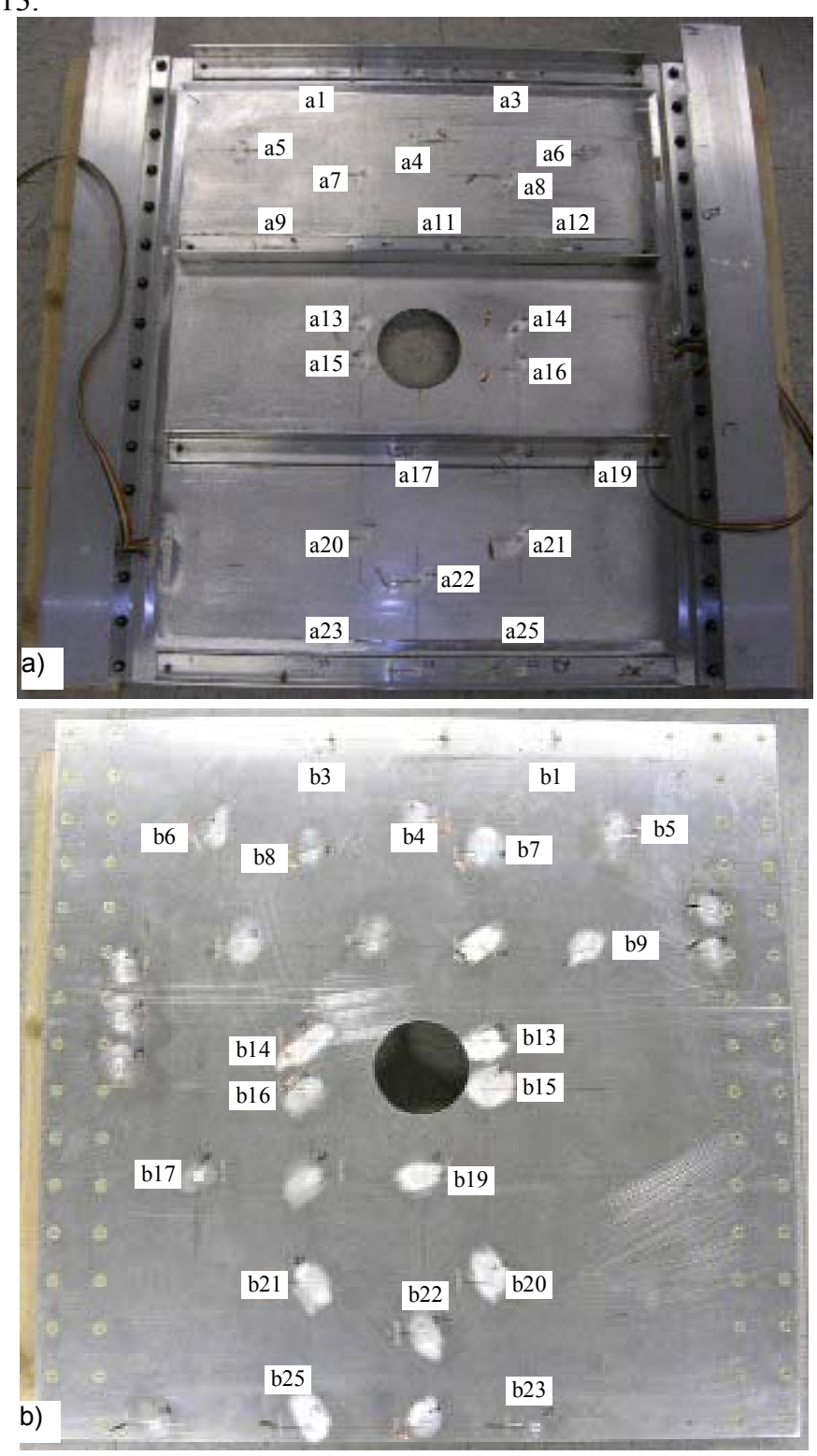

Figure 13 PWAS location on Panel 1 for the pulse-echo tests: (a) top face of Panel 1; (b) bottom face of Panel 1

The results shown in Figure 14 and Figure 15 refer to the disbond DB2. For this case the PWAS a7, a8, a20, and a21 were used. PWAS a7 is located close to the disbond DB2 whereas PWAS, a8, a20, and a21 are located in a pristine area where no damage is present. The comparison of the signal received from the damage with the signals where there is no damage is presented in Figure 15.

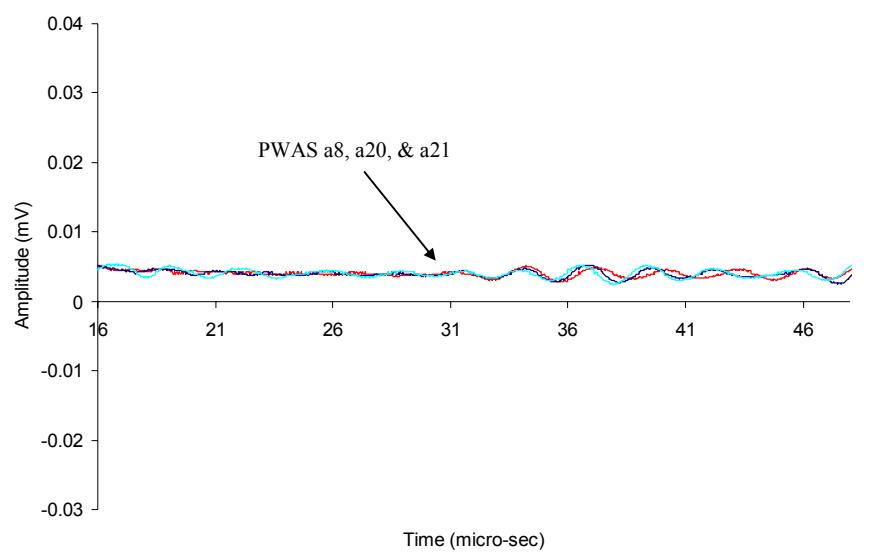

Figure 14 Pulse-echo method: pristine data from PWAS a8, a20, \& a21 showing a good consistent response

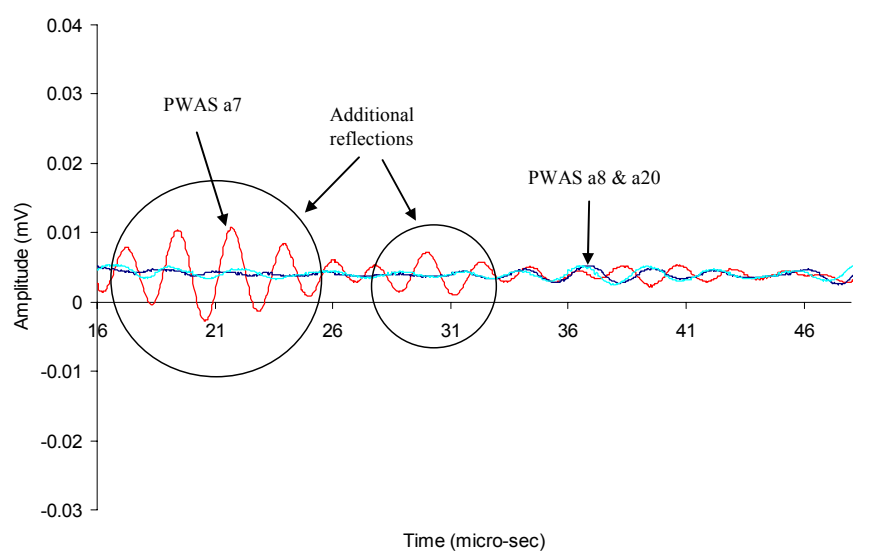

Figure 15 Pulse-echo method: initial bang removed, received signal at PWAS a7 from the disbond DB2. Pristine signals from PWAS a8 \& a20. Additional reflections due to the presence of damage

Figure 15 shows clear changes in the received signal (additional reflections) close to the damage. The presence of additional reflections is associated with echoes from the disbonded area.

\section{Embedded Ultrasonic Structural Radar (EUSR)}

The PWAS used for the Embedded Ultrasonic Structural Radar method and the actual location of the sensors on Panel 1 is presented in Figure 16. 


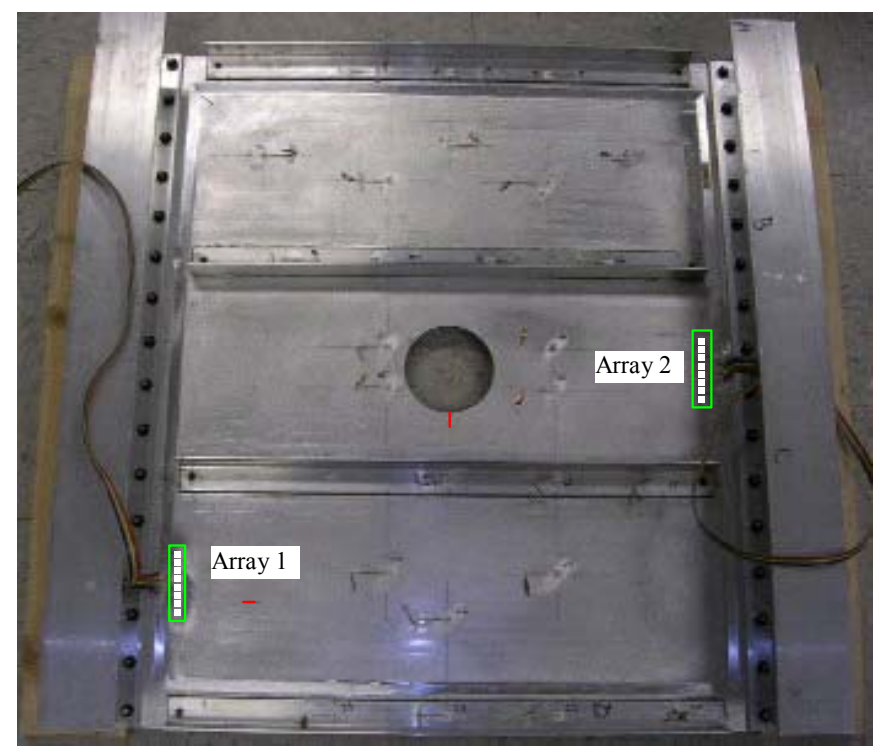

Figure 16 PWAS location on Panel 1 for the EUSR tests

Two phased arrays have been mounted on the top face of Panel 1. Each array consists of eight PWAS spaced at $1 \mathrm{~mm}$ interval. The excitation frequency used in the experiment was $330 \mathrm{kHz}$ and the calculated group velocity was $5000 \mathrm{~mm} / \mathrm{s}$. After the data was collected from the sensors, the parallel algorithm was used to construct the time-frequency domain signal. Next the $330 \mathrm{kHz}$ component was extracted using continuous wavelet transform. The Hilbert transform was applied to the filtered signal to get its envelope. In the A-scan image in Figure 17 and Figure 18, the blue curves are the continuous wavelet transform (CWT) filtered signals at the dial indicated direction, and the red curves are the envelopes of the respective signals. The 2-D images show the inspection result of the specific PWAS array.

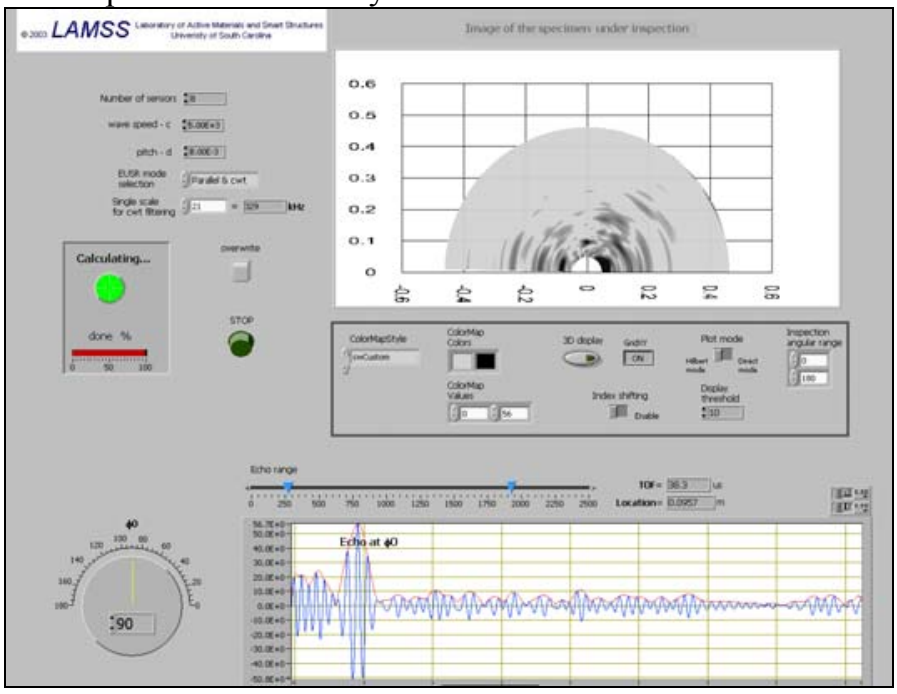

Figure 17 EUSR inspection result of the array \#1, Plate 1

The location of the crack with respect to Array \#1 is 50 $\mathrm{mm}$ perpendicular to the array which is the worst case possible because the echo from such a crack is equivalent with the echo of a hole with the diameter equal with the width of the slit, in this case 0.005 in or $0.13 \mathrm{~mm}$. Therefore it is very hard to distinguish the echo on the 2-D plot in Figure 17.

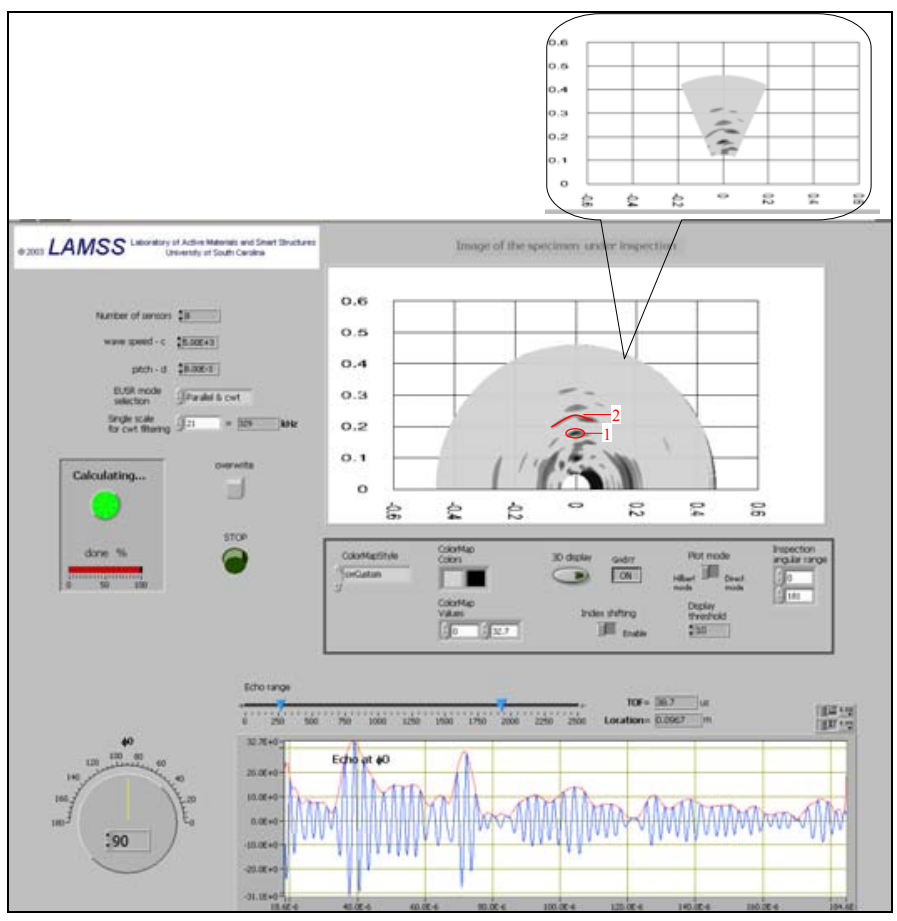

Figure 18 EUSR inspection result of array \#2, Plate 1

The location of the second damage with respect to Array \#2 is $170 \mathrm{~mm}$ parallel to the array. The half circumference of the middle hole will generate a strong reflection labeled as 1 in Figure 18. The center line of the circle is $220-\mathrm{mm}$ away from the array and the circle is symmetric to the perpendicular center line of both the circle and the array. From the asymmetric image labeled as 2 in Figure 18, we can generally tell there may be some disturbance resulting from damage around the circumference at the center line.

\section{Electromechanical Impedance method}

The PWAS used for the EMI method and the actual location of the sensors used on Panel 1 is presented in Figure 19.

The electromechanical impedance method (EM) was used to detect disbonds, cracks and corrosions. The results are presented in Figure 20 through Figure 22. The impedance spectrum from PWAS a1, a2, and a3 is presented in Figure 20. It can be seen that the resonant spectrums of the signals from PWAS a1 and a3 located on an area with good bond are almost identical. The resonant spectrum from PWAS a2 located on the disbond DB1 is very different showing new strong resonant peaks associated with the presence of the disbond.

The electromechanical impedance method also showed good results in detecting corrosion. In Figure 21 the resonant spectrum for the sensors PWAS b30 (corroded area) and PWAS b31 (undamaged area) are presented. The shift in the resonant frequency peak for the PWAS b30 is very clear which is an indication of a structural change due to the corrosion. The same phenomena of shifted and prominent resonant frequencies is noticeable in the crack CK1 detection shown in Figure 22. PWAS a29 and PWAS a31 show a consistent resonant spectrum and PWAS a30 presents the features mentioned above. 

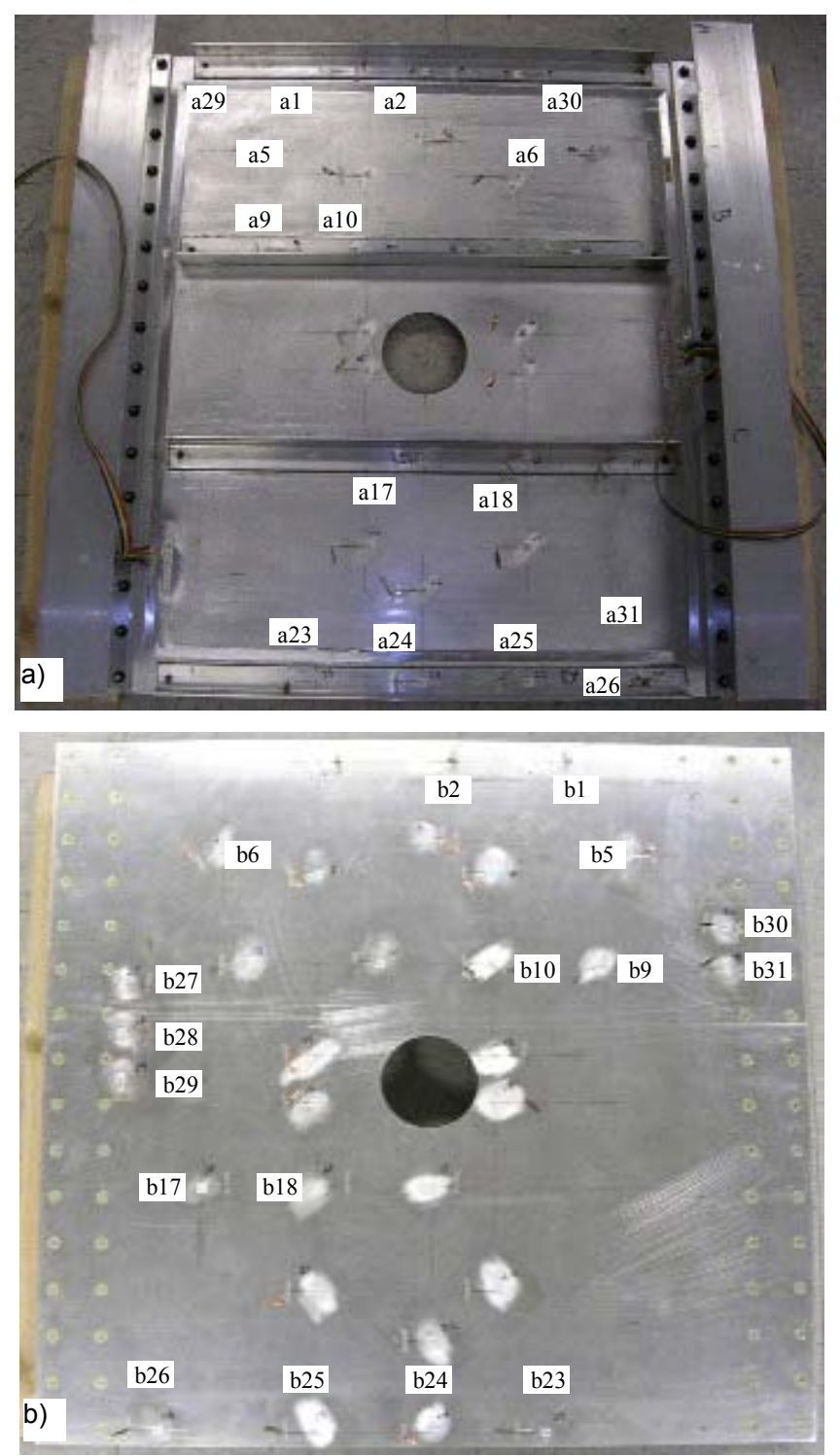

Figure 19 PWAS location on Panel 1 for the EM impedance tests: (a) top face of Panel 1; (b) bottom face of Panel 1

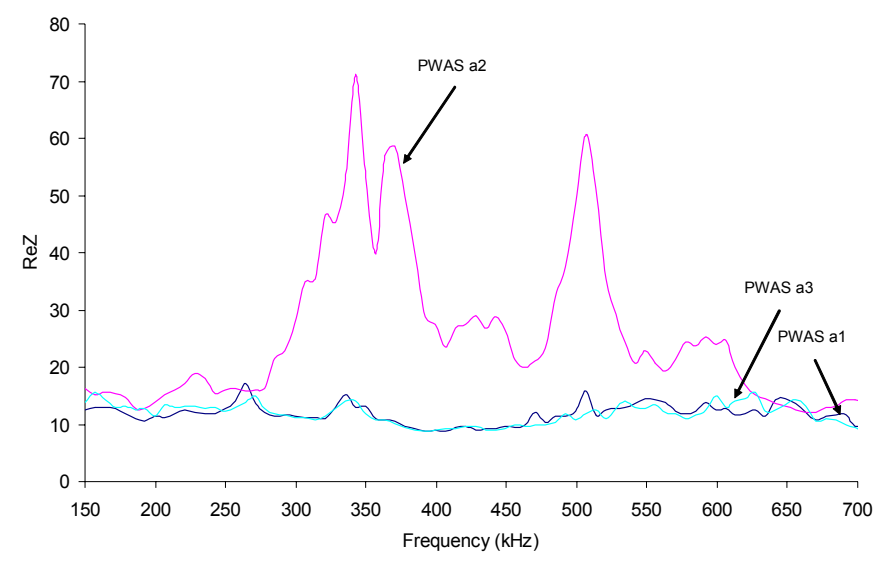

Figure 20 EM Impedance method: resonant frequencies spectrum showing increased amplitude for the signal received at the sensor located on the top of disbond DB1 (PWAS a2)

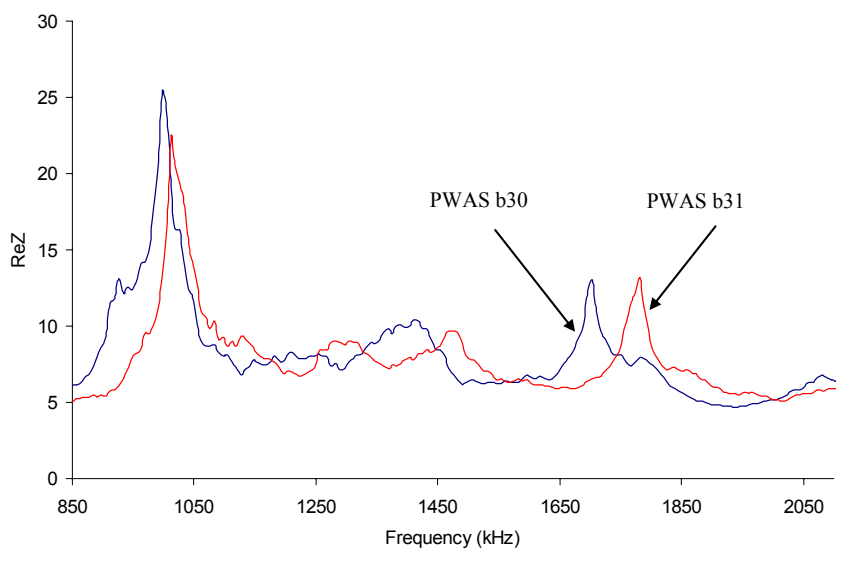

Figure 21 EM Impedance method: resonant frequencies showing shifted peaks for corroded area CR1 (PWAS b30) vs. undamaged area (PWAS b31)

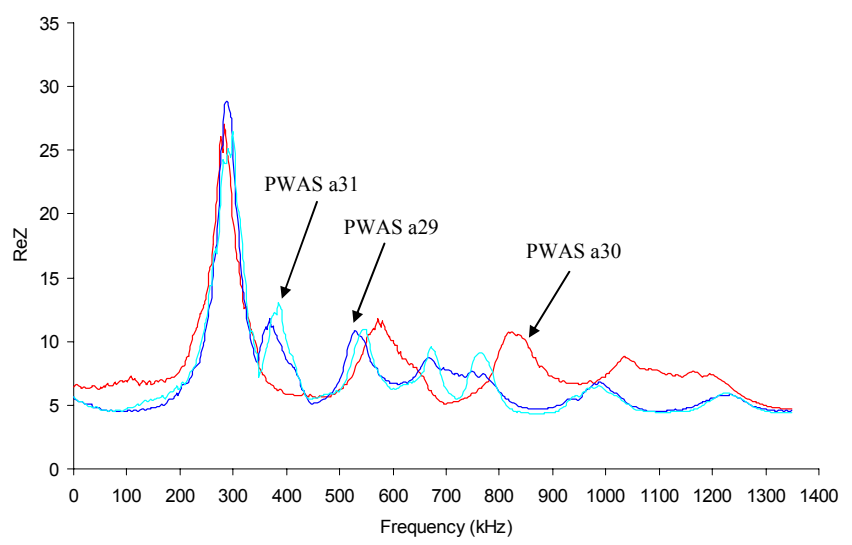

Figure 22 EM Impedance method: resonant frequencies of the sensor close to the crack CK1 (PWAS a30) and the sensors in a pristine area (PWAS b29 and b34)

\section{CONCLUSION}

In this paper, experimental investigations have been developed to demonstrate the possibility of using a structural health monitoring system based on piezoelectric wafer active sensors (PWAS). This work has been conducted in collaboration with NextGen Aeronautics, Inc. who provided the funding for the research and was responsible for the manufacturing of the two aluminum spacecraft panels.

The objective of this work was to show feasibility of key technologies applied to realistic structural components. Wave propagation methods were used for detection of cracks, corrosion and disbonds in stiffened metallic panels. Also, the ability to detect cracks under bolts and rivets was investigated. From the tests performed on the two metallic panels, an initial indication on how the detection methods compared are summarized in Table 4. 
Table 4 Summary of PWAS detection methods

\begin{tabular}{|l|ccc|c|}
\hline \multicolumn{1}{|c|}{ Damage } & \multicolumn{3}{|c|}{ Wave propagation } & $\begin{array}{c}\text { Standing } \\
\text { wype }\end{array}$ \\
& $\begin{array}{c}\text { Pitch- } \\
\text { catch }\end{array}$ & $\begin{array}{c}\text { Pulse- } \\
\text { echo }\end{array}$ & EUSR & $\begin{array}{c}\text { EM } \\
\text { Impedance }\end{array}$ \\
\hline Disbond & Fair & Good & $*$ & Good \\
Cracks & $*$ & Fair & Good & Fair \\
Corrosion & $*$ & $*$ & $*$ & Good \\
Crack under bolt & Good & Fair & $*$ & Fair \\
\hline
\end{tabular}

The results are based on near-field damage detection for a specific damage size. They allow us to conclude that successful damage detection can be achieved using wave propagation methods as well as the electromechanical impedance method.

\section{ACKNOWLEDGMENTS}

The financial support of NASA STTR program through Phase I topic T7-02 is gratefully acknowledged.

\section{REFERENCES}

1. Giurgiutiu, V.; Redmond, J.; Roach, D.; Rackow, K. (2000)"Active sensors for health monitoring of aging aerospace structures", Proceedings of the SPIE Conference on Smart Structures and Integrated Systems, Vol. 3985, pp. 294-305, Newport Beach, 2000

2. Giurgiutiu, V.; Zagrai, A. (2000) "Characterization of piezoelectric wafer active sensors", Journal of Intelligent Material Systems and Structures, Vol. 11, pp. 959-976, 2000

3. Moulin, E.; Assaad, J.; Delebarre, C.; Kaczmarek, H.; Balageas, D. (1997) "Piezoelectric Transducers Embedded in a Composite Plate: Application to Lamb Wave Generation", Journal of Applied Physics, Vol. 82, No. 5, 1997, pp. 2049-2055

4. Lin, X.; Yuan, F. G. (2001a) "Diagnostic Lamb Waves in an Integrated Piezoelectric Sensor/Actuator Plate: Analytical and Experimental Studies", Smart Materials and Structures, Vol. 10, 2001, pp. 907-913

5. Liu, T.; Veidt, M.; Kitipornchai, S. (2003) "Modeling the Inputoutput Behavior of Piezoelectric Structural Health Monitoring systems for Composites Plates", Smart Materials and Structures, Vol. 12, pp. 836-844, 2003

6. Lee, B.C.; Staszewski, W. J. (2003) "Modeling of Lamb waves for damage detection in metallic structures: Part I Wave propagation", Smart Materials and Structures, Vol. 12, pp. 804814, 2003

7. Lee, B. C.; Staszewski, W. J. (2003) "Modeling of Lamb waves for damage detection in metallic structures: Part II Wave interactions with damage", Smart Materials and Structures, Vol. 12 , pp. 804-814, 2003

8. Qing, X.; Beard, S. J.; Kumar, A. (2003) "Optimal Placement of Sensor/Actuator in SMART Layer for Structural Health Monitoring", Structural Health Monitoring - An International Journal (in press)

9. Giurgiutiu, V. (2003) "Lamb Wave Generation with Piezoelectric Wafer Active Sensors for Structural Health Monitoring", SPIE's 10th Annual International Symposium on Smart Structures and Materials and 8th Annual International Symposium on NDE for Health Monitoring and Diagnostics, 2-6 March 2003, San Diego, CA, paper \# 5056-17 